\title{
Projected Area Based 3D Shape Similarity Evaluation
}

\author{
Tetsuo Miyake $^{1}$, Naoya Iwata ${ }^{2}$, Satoshi Horihata ${ }^{1}$, and Zhong Zhang ${ }^{1}$ \\ ${ }^{1}$ Dept. of Prod. Sys. Eng., Toyohashi University of Technology, \\ 1-1, Hibarigaoka, Tenpaku, Toyohashi, 441-8580, Japan \\ 2 Toyohashi University of Technology. Now in Aishin AW Co., LTD, \\ 10, Takane, Fujii-cho, Anjo City, Aichi 444-1192, Japan \\ \{miyake, horihata, zhang\}@is.pse.tut.ac.jp
}

\begin{abstract}
Because the appearance of 3D objects changes according to viewing directions, it is not easy to evaluate similarity between two objects in a few appearances. In this paper we propose similarity measure between two shapes of $3 \mathrm{D}$ objects. The feature of a shape is represented by a distribution of a projected area on a unit sphere, and the distribution is expanded in spherical harmonics. The degree of similarity between several kinds of shape is calculated and is compared with human sense. The results of computer simulation demonstrate the validity of our method.
\end{abstract}

\section{Introduction}

We recognize a 3D shape of an object and can evaluate similarity between two objects without much difficulty. For example, we say on occasion that this cup is similar to that. But if we are asked which body a cylinder or a pyramid is similar to a prism, what answer shall we return? Generally speaking, because the appearance of an object changes according to viewing directions, it is not easy to evaluate similarity between two objects in a few appearances. Even if two objects are exactly same, it is hard to draw the right conclusion at a glance.

Many methods are proposed in the published papers that have addressed shape similarity evaluation for shape searching [1. Recently similarity search between a query object and a target in a database is actively studied in data retrieval of $3 \mathrm{D}$ objects. On the other hand, it is well known that expansion into a set of orthonormal basis is strong means in analyzing a given function, and spherical harmonics has performed the same role in some of these studies. Our proposed method also uses a set of spherical harmonics, but it is some different in defining a function that expresses the feature of a shape of an object.

In order to retrieve a target object with high reliability, a shape-based search method must be presented. There are various kinds of 3D structural model including CAD models, voxel data and polygonal meshes in the previous works that applied orthogonal expansion to similarity evaluation. Princeton Shape Retrieval and Analysis Group have got successful results using several structural models [2, 3, 4. Saupe et al. developed a method that was applied to a polygonal mesh model 55. Proriol tried shape recognition using one or more than 
one silhouettes of simple bodies [6]. The body with a certain solid structure was needed in calculating expansion coefficients. Tanaka et al. represented polyhedral objects using extended Gaussian images 7 .

The aim of our study is not to retrieve an object that is most similar to the given object, but to define a similarity measure that simulate a human sense of the similarity evaluation. If we can realize it, we can clarify what kind of feature of appearance a human perceives in evaluating shape similarity. It is not difficult in a sense to judge whether or not a query shape is equal to or similar to a target using detailed geometrical description. However, in practice, we usually have no structural information about an object's shape except its appearances. Therefore we define the feature function of an object's shape based on a projected area, a silhouette of an object, which must be one of the simplest feature of an object.

In this paper, we first define similarity measure for evaluating the 3D shape of an object. The measure is determined based on the spherical harmonics. Next we evaluate the similarity of several kinds of shape and finally we compare the measure with human sense. The results of computer simulation demonstrate the validity of our method.

\section{Principle of Similarity Evaluation}

It is necessary to solve the following problems so as to perform similarity evaluation.

- What feature of an object in appearance is observed.

- How viewing directions are determined.

- How the observed features are transformed to a numerical value.

- How the numerical values are analyzed.

We discuss each problem in the following sections.

\subsection{Observation of the Object}

The object is assumed to be placed at the center of a sphere. We observe the object from a point on the surface of a unit sphere, which position is expressed by $(\theta, \phi)$. Figure 1 shows the object and the viewpoint on the surface. Since the appearance of an object changes according to the viewpoint, we can not determine its 3D shape based only on one appearance. Therefore we need to observe it from not a few number of viewpoints.

It is preferable to observe the object in the directions uniformly distributed in space, but it is known that such directions can not be realized. Though the vertices of regular polyhedra can be applied, the maximum number is at most 20 . In this paper we determine the direction where the object is observed using a geodesic dome. The geodesic dome is one solution for generating almost uniformly distributed directions [8]. Initially the dome has a shape of a regular icosahedron. Each facet of the polyhedron, whose shape is a triangle, is iteratively divided as shown in Fig. 2. After the facet is divided two times, 162 vertices are generated on the spherical surface. 


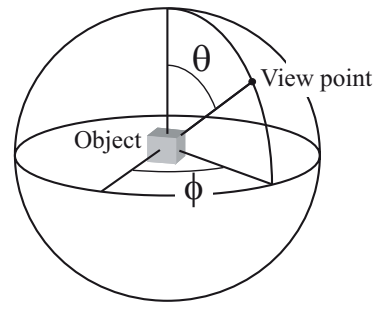

Fig. 1. The object is observed from $(\theta, \phi)$

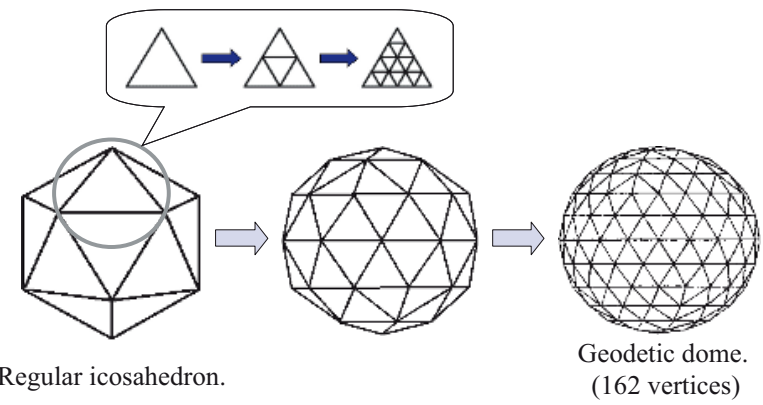

Fig. 2. Geodesic dome is generated based on a regular icosahedron

\section{$2.2 \quad$ Feature of the Object}

Several kinds of feature including colors, textures, a number of vertices and outline shapes are observed in the projected image of the object. Because of their change in appearance according to the viewing direction, it is not easy to compare two objects and judge whether they are similar or not.

For doing that, we propose to use a projected area of the image as a clue of the similarity measure. Suppose that we can only observe a silhouette of an unknown object. In spite of the particular condition, we conclude that the shape of the object must be a sphere if the image projected to any direction is a circle, for example. Therefore it is considered that the shape similarity can be evaluated using the distributed function of the projected area on a spherical surface.

\subsection{Similarity Measure}

Spherical harmonics are strong means for analyzing a function $f(\theta, \phi)$ defined on a unit sphere. It is to the function what a trigonometric function is to a single-valued function with respect to time.

Spherical harmonics denoted $Y_{l, m}$ is defined as

$$
Y_{l, m}(\theta, \phi)=\sqrt{\frac{2 l+1}{4 \pi} \frac{(l-m) !}{(l+m) !}} P_{l}^{m}(\cos \theta) e^{i m \phi} .
$$


$P_{l}^{m}(x)$ is the associated Legendre functions expressed by

$$
P_{l}^{m}(x)=\left(1-x^{2}\right)^{m / 2} \sum_{k=0}^{[(l-m) / 2]} \frac{(-1)^{k}}{2^{k} k !} \frac{(2 l-2 k-1) ! !}{(l-m-2 k) !} x^{l-m-2 k},
$$

where $l$ and $m$ are integer numbers with $0 \leq|m| \leq l$. In this paper, we define similarity measure based on real spherical harmonics, defined as

$$
\begin{aligned}
\tilde{Y}_{l, 0}(\theta, \phi) & =\sqrt{\frac{2 l+1}{4 \pi}} P_{l}^{0}(\cos \theta), \\
\tilde{Y}_{l, m}(\theta, \phi) & =\sqrt{\frac{2 l+1}{2 \pi} \frac{(l-m) !}{(l+m) !}} P_{l}^{m}(\cos \theta) \cos m \phi, \\
\tilde{Y}_{l,-m}(\theta, \phi) & =\sqrt{\frac{2 l+1}{2 \pi} \frac{(l-m) !}{(l+m) !}} P_{l}^{m}(\cos \theta) \sin m \phi .
\end{aligned}
$$

The square integrable function $f(\theta, \phi)$ on a unit spherical surface is expanded to

$$
f(\theta, \phi) \sim \sum_{l=0}^{\infty} \sum_{m=-l}^{l} a_{l, m} \tilde{Y}_{l, m}(\theta, \phi),
$$

in terms of completeness of the spherical harmonics, where $a_{l, m}$ is a coefficient of the expansion expressed by

$$
a_{l, m}=\int_{0}^{2 \pi} d \phi \int_{0}^{\pi} \tilde{Y}_{l, m}(\theta, \phi) f(\theta, \phi) \sin \theta d \theta .
$$

When calculating (4), the equation is discretized to

$$
a_{l, m}=\sum_{i=1}^{N} \tilde{Y}_{l, m}(\theta, \phi) f(\theta, \phi) \Delta A
$$

where $N$ is the number of view points and $\Delta A$ is an infinitesimal area at each view point $(\theta, \phi)$. The value is equal to a surface area of a unit sphere divided by $N$, then $\Delta A=4 \pi / N$. Since these coefficients are unique to the function $f(\theta, \phi)$, the difference of the coefficients can be used for the similarity measure.

We should note, however, that distribution of $f(\theta, \phi)$ changes according to the pose of an object and as a matter of course the coefficients change. Therefore we consider all coefficients together that have a same number of degree $l$ so as to get rid of the effect of the pose. Letting

$$
\boldsymbol{v}^{(l)}=\left[a_{l, l} a_{l, l-1} \cdots a_{l,-l}\right]
$$

define a coefficient vector $\boldsymbol{v}^{(l)}$, the norm of $\boldsymbol{v}^{(l)}$ is represented by

$$
\left\|\boldsymbol{v}^{(l)}\right\|=\sqrt{\sum_{k=-l}^{l} a_{l, k}^{2}}
$$


Though the vector changes according to the pose, its norm does not [7].

Finally we construct a feature vector $s$ such that

$$
\boldsymbol{s}=\left[c^{(0)} c^{(1)} \cdots c^{(l)} \cdots\right],
$$

where each component $c^{(l)}$ is expressed by

$$
c^{(l)}=\frac{\left\|\boldsymbol{v}^{(l)}\right\|}{\sqrt{\sum_{k=0}^{\infty}\left\|\boldsymbol{v}^{(k)}\right\|^{2}}} .
$$

Because each value of $\left\|\boldsymbol{v}^{(l)}\right\|$ depends on the size of the image, normalization is then necessary. By normalization, all geometrically similar shapes can have the same feature vector $s$ in (8). It is thought that $s$ represents the feature of the shape.

The degree of similarity, denoted $S_{A, B}$, between the object $A$ and $B$ is calculated by

$$
S_{A, B}=s_{A} \cdot s_{B}=\sum_{k=0}^{\infty} c_{A}^{(k)} c_{B}^{(k)}
$$

where $c_{X}^{(k)}$ represents the $k$-th component of the feature vector $s_{X}$. Since (10) means a natural inner product, it is obvious that $0.0<S_{A, B} \leq 1.0$. The value of $c^{(0)}$ is based on the normalized value of $a_{0,0}$, which is the integral of $f(\theta, \phi)$ over the surface. Therefore $0.0<c_{A}^{(0)}$ and $0.0<c_{B}^{(0)}$, and it always holds that $0.0<S_{A, B}$. If and only if the two objects are exactly the same from the view point of its silhouette, except the mirror symmetry, $S_{A, B}$ is equal to 1 .

\section{$3 \quad$ Numerical Simulation of Similarity Evaluation}

Numerical simulation of similarity evaluation is conducted using virtual objects defined in a computer. Firstly it is demonstrated that the proposed similarity measure is pose invariant. Secondary the degree of similarity is evaluated between two objects with various kinds of shape.

\subsection{Generation of Distribution Function}

We use polyhedra and quadric surfaces as objects in the similarity evaluation. These shape models are convenient for mathematical treatment in the computer and can represent complicated shapes and curved surfaces. The polyhedral object is defined by boundary representation.

The object is placed at the center of the geodesic dome such that the center of gravity of it coincides with the center. If the center of gravity is outside of the object such as a torus, no problem occurs in the evaluation. One of the vertices of the dome is chosen as a viewpoint and the projected image of the object is generated on a virtual image plane. The projection is assumed to be parallel to 
the line from the center to the viewpoint and the image plane is assumed to be perpendicular to the line. Then the projected area of the image is calculated. By calculating the areas at all viewpoints in the half region of the dome, the distributed function of the projected area on a unit sphere is generated.

\subsection{Pose Invariance}

The similarity measure must satisfy pose invariance. It means that if the object $A$ is exactly the same as $B$ but its pose is different from $B$ as shown in Fig. 3, the degree of similarity $S_{A, B}$ must become 1.0.
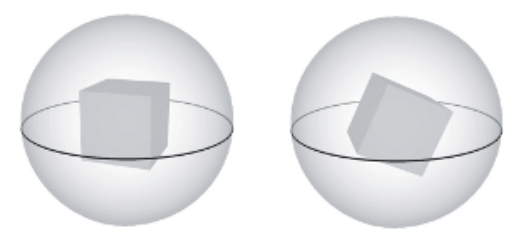

Fig. 3. The same two objects in the different pose

We evaluated the similarity between the same two objects in the different pose using three polyhedra, one convex and two concave. These bodies are shown in Fig. 4 A cube that belongs among a convex polyhedron has no occlusion by itself, but a concave polyhedron has. The projected area of the thin body changes much according to the viewing direction.

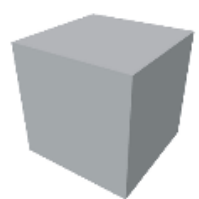

(a) A cube.

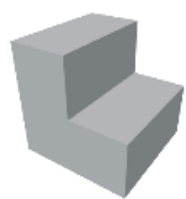

(b) A cube-like body.

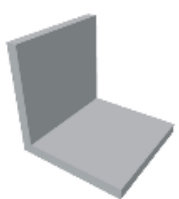

(c) A thin body.

Fig. 4. Three polyhedra; (a) is convex and (b) and (c) are concave

A body to be compared was turned on the vertical and the horizontal axis, and was compared to the original one. The results of similarity evaluation for the thin body are shown in Fig. 5 . Here the maximum degree of expansion in spherical harmonics is limited to 10. It can be seen that the degree of similarity $S_{A, A}$ is always 1.0 irrespective of its pose. Because almost the same results were obtained using the other bodies, it is concluded that the proposed similarity evaluation satisfies pose invariance. 


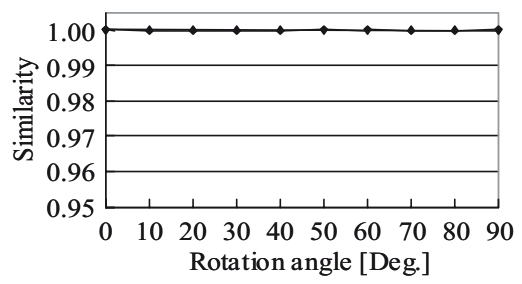

(a) Turned on the vertical axis.

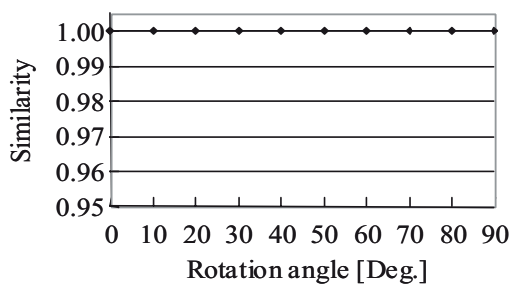

(b) Turned on the horizontal axis.

Fig. 5. The results of similarity evaluation according to the pose change

\subsection{Similarity Evaluation}

We evaluate the degree of similarity between two objects among twelve bodies shown in Fig. 6. These bodies are divided into three types of shape according to its appearance; a convex shape, a concave shape and a concave and thin shape. The shape of all bodies is defined by the boundary between inside and outside.

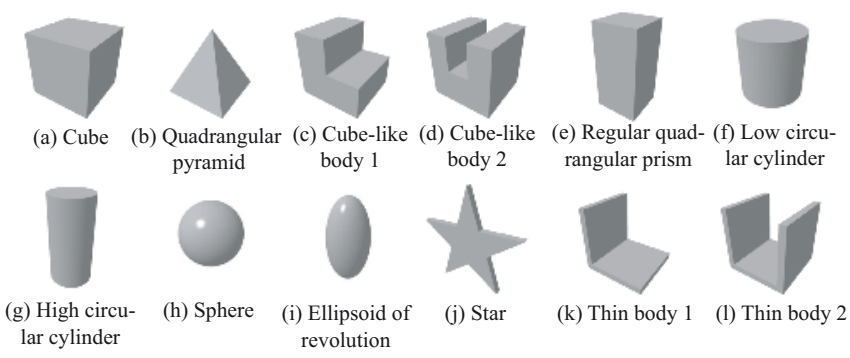

Fig. 6. Twelve bodies used in the similarity evaluation

Figure 7 shows the result of similarity evaluation. Each gray patch represents the degree of similarity between the two objects in the row and column position. Lighter gray means that the degree is higher.

It can be seen from the light and dark gray distribution that the bodies are divided into thick and thin ones. The degree of similarity of almost all bodies against a star is lowest. Since the star has almost a $2 \mathrm{D}$ shape compared with the other bodies, it is considered that the distribution pattern of the projected area of the star is much different.

Figure 8 shows the bodies similar to a cube and similar to a star in order of the degree of similarity from highest to lowest. The degree of similarity of a thick body to a thin body is low and the reverse is also true. It can be said that the similarity measure represents the feature of the shape well. It is a little strange from the point of view of human sense that the ellipsoid of revolution has lower degree than the thin body 2 in Fig. 8(a). On the other hand, the order shown in fig. 8 (b) is fully understandable. 


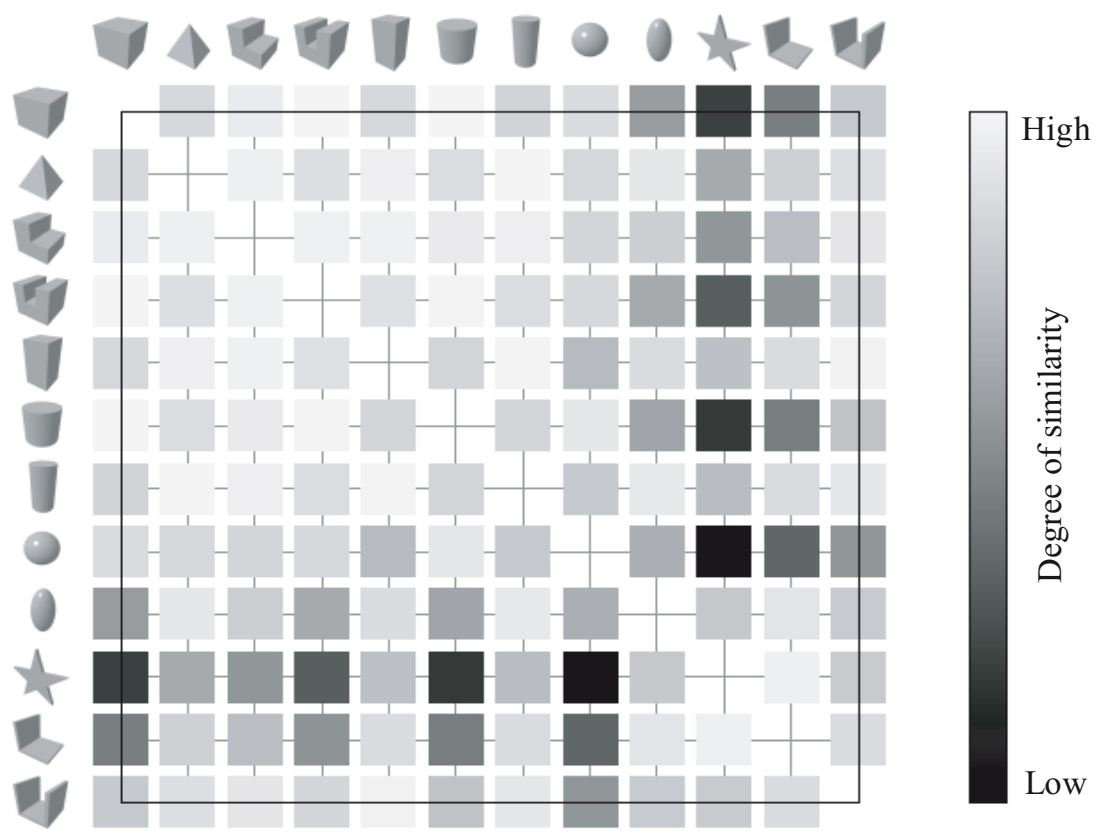

Fig. 7. The result of similarity evaluation

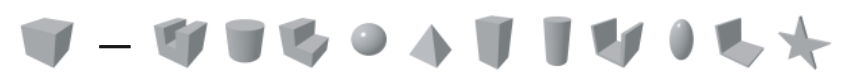

(a) Between a cube and other bodies.

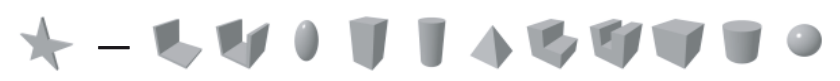

(b) Between a star and other bodies.

Fig. 8. The order according to the degree of similarity

It may seem curious that though a star indicates the lowest degree of similarity to a cube, a sphere to a star. But because the feature vector defined by (8) is a 11-dimensional vector, the result is possible. Calculating an inner product of the two 11-dimensional feature vectors for the similarity evaluation means that the degree of similarity is measured by the angle between two vectors, which has one dimension.

Figure 9 shows the shape similarity in two dimensions. The vertical axis indicates the degree of similarity to a sphere, and the horizontal axis to a circle. The figure shows that an ellipsoid of revolution is similar both to a sphere and to a circle, which expresses the characteristic of its shape properly. 


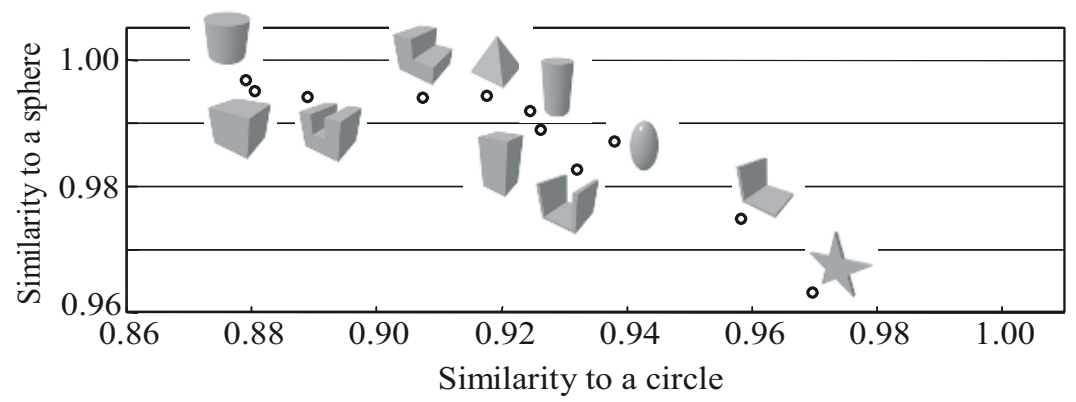

Fig. 9. 2D representation of the degree of similarity

\section{Comparison with Human Sense}

Human sense in shape similarity evaluation was investigated through a questionnaire survey. The survey was conducted on the internet web site and 127 visitors answered the question whether a cube is similar to a pyramid, a cylinder and so on. Figure 10(a) shows the percentage of the number of who answered "yes", and Fig. 10 (b) shows the results of our method. The eight bodies arranged in descending order of the percentage and the similarity measure are shown in Figure 11(a) and (b), respectively.

The proposed similarity measure agrees well with human sense except for a regular quadrangular prism. Since a cube and a regular prism have only straight line edges and they resemble each other from the point of view of topological geometry, it is natural for a human to feel that a prism is similar to a cube, even if the height and width ratio is different. Information about whether the body is convex or concave, or whether it has plain surfaces or curved ones gives us intuitive impression, and affects our sense of similarity evaluation.

We need to consider topology as well as geometry, but topology itself is hard to be perceived. A human stores much knowledge about appearances of an object, and it is thought to be the reason why a human can describe its topological features. Object recognition may have to be done first before similarity evaluation

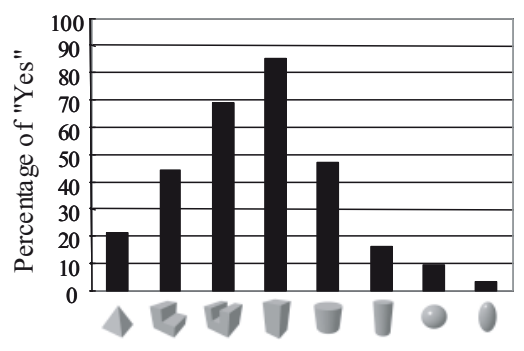

(a) Result of the questionnaire survey.

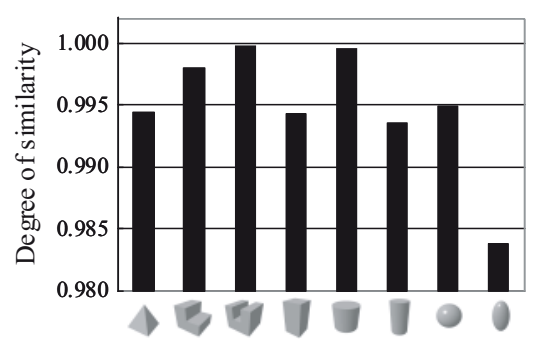

(b) Result of the proposed method.

Fig. 10. A cube is compared to the other shapes 


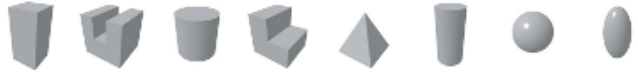 \\ (a) Result of the questionnaire survey.

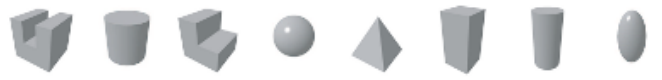 \\ (b) Result of the proposed method.}

Fig. 11. Eight bodies arranged in descending order of similarity to a cube

by the computer. Therefore we need to collect as much visible information as possible in order to simulate a human brain. The number of vertices, the length of inner edges or an outline and various kinds of 2D shape descriptors are valid features which can be defined in the same spherical function as the projected area.

\section{Conclusion}

We proposed 3D shape similarity evaluation using the projected area in multiple directions. A spherical function defined by the distribution of the projected area on a unit sphere is expanded in spherical harmonics, and the similarity measure is determined by coefficients of the expansion. The numerical simulation has demonstrated that the similarity measure well expresses the features of the shape of an object. After that the proposed measure has been compared with human sense through the questionnaire survey.

Finally the proposed measure almost agrees with human sense but some difference became clear. Though only the projected area was used as the feature of the object, the simulation has shown satisfactory results. It is considered that more information can reduce the present gap between the simulation and the human sense. The future study will include the distribution of the length of edges, which supply some topological information.

\section{References}

1. Iyer N., Jayanti S., Low K., Kalyanaraman Y., Ramani K.: Three-dimensional shape searching: state-of-the-art review and future trends. Computer Aided Design, 37 (2005) 509-530

2. Funkhouser T., Min P., Kazhdan M., Chen J., Halderman A., Dobkin D., Jacobs D.: A search engine for 3D models. ACM Trans. on Graphics, 22 (2003) 83-105

3. Kazhdan M., Funkhouser T., Rusinkiewicz S.; Rotation Invariant Spherical Harmonic Representation of 3D Shape Descriptors. Eurographics Symposium on Geometry Processing 1 (2003) 156-165

4. Princeton Shape Retrieval and Analysis Group: 3D Model Search Engine http://shape.cs.princeton.edu/search.html 
5. Saupe D. and Varanić D. V.: 3D model retrieval with spherical harmonics and moments. Proc. of DAGM2001 23 (2001) 392-397

6. Proriol J.: Definition of new 3D invariants. Intl. J. of Neural Systems 7 (1996) 709-714

7. Tanaka K, Sano M, Mukawa N, Kaneko H.: 3D object representation using spherical harmonic functions. Proc. of the IEEE/RSJ (1993) 1873-1880

8. Horn B. k. P.: Robot Vision. The MIT Press (1986) 